\title{
Characterization of the electrical and extracellular matrix remodeling in patients with HF: comparison between HEpEF and HErEF.
}

\author{
Gabriel da S. Ferreira*, Fernando B. Cardoso MD, Luis M. Silva BSc, Camilla Toledo BSc, Lígia M. Antunes- \\ Correa PhD, Vinicius C. Ribeiro MD, Thiago Q. A. C. Silva MD PhD, Layde R. Paim BSc, Gustavo Martins MD, \\ Wilson Nadruz MD PhD, Celso D. Ramos MD PhD, Andrei Sposito MD PhD, Jose R. Matos-Souza MD PhD, \\ Tomas G. Neilan MD MPH, Michael Jerosch-Herold PhD, Otávio R. Coelho-Filho MD PhD MPH
}

\begin{abstract}
This study aimed to assess and compare myocardical electrical and extracellular matrix remodeling in patients with heart failure (HF) with preserved ejection fraction (HFpEF) and with heart failure with reduced ejection fraction (HFrEF) using a contemporary multimodality approach. We recruited 47 individuals presenting HF (22 females, 25 males), NYHA classes II-III, stratified according to LVEF in $\operatorname{HFpEF}(n=23)$ and $\operatorname{HFrEF}(n=24)$. They underwent cardiac MRI (CMRI) including T1-mapping, echocardiography for global longitudinal strain (GLS), cardiopulmonary exercise test (CPET), cardiac sympathetic imaging with mIBG and biomarkers. Results show native-T1 and extracellular volume fraction (ECV) were not different between groups. The mIBG derived heart-to-mediastinum ratio (HMR) were reduced in both groups. Considering the entire cohort, as well as the HFrEF subgroup separately, ECV was inversely associated to HMR and to adjusted VO2 max, and positively associated to NT-proBNP, US-Troponin and to GLS. Considering the HFpEF subgroup separately, only ECV and GLS association remained significant. The study highlights that similar extracellular matrix remodeling, assessed by ECV, between both subgroups confirms diffuse fibrosis as part of the HFpEF cardiac phenotype, which may partially explain its unfavorable prognosis and limited response to antiremodeling therapies.
\end{abstract}

\section{Key words:}

Heart failure, Preserved ejection fraction, Cardiac remodeling

\section{Introduction}

Individuals with heart failure (HF) with preserved ejection fraction (HFpEF) experience high morbidity and mortality, but contrary to HF with reduced EF (HFrEF), anti-remodeling therapies have failed to reduce mortality. Current methods to detect LV reverse remodeling reveal primarily advanced disease and fail to detect tissue phenotypes of early-HF-stages.

We aimed to investigate and compare myocardial tissue remodeling in HFpEF and HFrEF using a contemporary multimodality approach to assess myocardial electrical and extracellular matrix remodeling.

\section{Results and Discussion}

Forty-seven individuals (age:54.1 \pm 11 years, BMI:30.5 \pm 6 , 22 females, mean-LVEF: $42.2 \pm 15 \%, 24 \mathrm{HFrEF}$ and 23 $\mathrm{HFpEF}$ ) were prospectively recruited. They were symptomatic HF patients (NYHA II-III) stratified according to LVEF in HFrEF $(<50 \%)$ and HFpEF $(>/=50 \%)$ and underwent cardiac MRI (CMRI) including T1-mapping, echocardiography for global longitudinal strain (GLS), cardiopulmonary exercise test (CPET), cardiac sympathetic imaging with $\mathrm{mIBG}$ and biomarkers. All individuals were recruited when stabilized using optimized HF therapy. As expected LVEF was different between groups ( $32 \pm 8.5 \% \mathrm{vs} .58 .2 \pm 7 \%, p<0.001)$ and the adjusted-VO2max was more reduced in HFrEF (18.3 \pm 4.7 vs. $22.8 \pm 5.2 \mathrm{ml} / \mathrm{min} / \mathrm{kg}, \mathrm{p}=0.01)$. While $\mathrm{GLS}$ was reduced in HFrEF compared to HFpEF (HFrEF:-8.2 \pm 3.7 \%vs. HFpEF:- $15.2 \pm 3.7 \%, p<0.001$ ), both the native-T1 (HFrEF:1101.6 \pm 213 vs. HFpEF:1146 $\pm 58, \mathrm{p}=0.4$ ) and extracellular volume fraction (ECV), though abnormally high, were not different among HF groups (HFrEF:0.36 \pm
0.07 vs. HFpEF:0.33 $\pm 0.03, p=0.06)$. The mIBG derived heart-to-mediastinum ratio (HMR) were also reduced in both groups but more evident in the HFrEF $(1.44 \pm 0.17$ vs. $1.62 \pm 0.21, p=0.007)$. Considering the entire cohort, ECV was inversely associated to HMR $(r=-0.45, p=0.023)$ and to adjusted-VO2max $(r=-0.41, \quad \mathrm{p}=0.02) ;$ and positively associated to NT-proBNP $(r=0.52, p<0.001)$, US-Troponin $(r=0.6, p=0.009)$ and to GLS $(r=0.59$, $\mathrm{p}<0.001)$. While all these associations were maintained in HFrEF, only the association of ECV and GLS remained significant $(r=0.7, p<0.05)$ in the HFpEF subgroup.

\section{Conclusions}

This study highlights the considerable myocardial tissue remodeling present in patients with HFpEF. Extracellular matrix remodeling, assessed by ECV, was similar in $\mathrm{HEpEF}$ and HErEF, confirming that diffuse fibrosis is part of the HFpEF cardiac phenotype, which may partially explain its unfavorable prognosis and limited response to anti-remodeling therapies seen in contemporary clinical trials.

\section{Acknowledgement}

This research was supported by CNPq, Fapesp and FAEPEX.

Lorell BH, Carabello BA. Left ventricular hypertrophy: pathogenesis, detection, and prognosis. Circulation. 2000;102(4):470-9.

Boluyt MO, O'Neill L, Meredith AL, Bing OH, Brooks WW, Conrad CH, et al Alterations in cardiac gene expression during the transition from stable hypertrophy to heart failure. Marked upregulation of genes encoding extracellular matrix components. Circulation research. 1994;75(1):23-32. 